\title{
Clinical features and risk factors for blood stream infections of Candida in neonates
}

\author{
MINGYUE LIU, SIYUAN HUANG, LINYING GUO, HONGRI LI, \\ FEI WANG, QI ZHANG and GUOWEI SONG
}

Department of Critical Medical Care, Affiliated Children's Hospital, Capital Institute of Pediatrics, Beijing 100020, P.R. China

Received August 24, 2014; Accepted May 29, 2015

DOI: $10.3892 / \mathrm{etm} .2015 .2626$

\begin{abstract}
Candida species are the leading cause of invasive fungal infections in children admitted to hospital. However, few data exist with regard to the clinical features, risk factors and prognosis for candidemia in neonates. The present retrospective study included 40 neonates from the Affiliated Children's Hospital of the Capital Institute of Pediatrics (Beijing, China) in the time period between January 1, 2006 and December 31, 2010 (candidemia group, $\mathrm{n}=19$; non-candidemia group, $\mathrm{n}=21$ ). The clinical characteristics, prognosis and previously identified risk factors for the two groups were recorded. According to the forward stepwise multivariate logistic regression analysis, administration of antibiotics $>2$ weeks prior, the use of glycopeptide antibiotics, maternal candidal vaginitis and secondary gastrointestinal surgery were identified as predictors of candidiasis. When compared with the non-gastrointestinal dysfunction group, the proportion of neonates that had been subjected to parenteral nutrition, central venous catheters, gastrointestinal surgery, secondary gastrointestinal surgery, repeated tracheal intubation and glycopeptide antibiotic administration was significantly higher in the gastrointestinal dysfunction group $(\mathrm{P}<0.05)$. Long-term application of antibiotics, use of glycopeptide antibiotics, maternal candidal vaginitis and secondary gastrointestinal surgery appeared to be the risk factors of candidemia in neonates. The neonates co-existed with gastrointestinal dysfunction suffering from candidemia were likely to experience growth retardation at 6 months after hospital discharge. Candidemia is potentially life-threatening situation for neonates, and if patients do not succumb it may affect their early development.
\end{abstract}

Correspondence to: Dr Qi Zhang, Department of Critical Medical Care, Affiliated Children's Hospital, Capital Institute of Pediatrics, 2 Yabao Road, Beijing 100020, P.R. China

E-mail: qizhangcn@yeah.net

Key words: neonatal, candidemia, clinical features, risk factors, prognosis, gastrointestinal dysfunction

\section{Introduction}

Invasive fungal infections are a considerable cause of morbidity, mortality, increased hospital stay durations, and high health care costs in critically ill or immunocompromised children (1-4). The majority of invasive fungal infections in children occur in a hospital setting, and the majority of infections are caused by Candida species $(1,5)$. In addition to the presence of Candida species in the blood (candidemia), Candida infections can disseminate to each main organ, including the brain, lung, liver, heart, kidneys, eyes and spleen $(2,3)$. As neonates have immature specific and nonspecific immune functions, they are more vulnerable to invasion by Candida species compared with adults and children. The signs and symptoms of Candida infection are severe and may resemble sepsis syndrome $(2,4)$, so early diagnosis is crucial, although it remains challenging $(6,7)$. Therefore, the aim of the present study was to summarize the clinical characteristics of candidemia in neonates, and determine whether specific risk factors may be identified to aid the selection of patients who are more likely to develop candidemia. Understanding the risk factors, clinical features and complications affecting the prognosis of candidemia in the neonatal period may help pediatricians to improve their protective medical measures and more effectively treat neonatal candidemia.

In addition, gastrointestinal dysfunction is common in neonates with congenital gastrointestinal dysfunction (such as gastrointestinal atresia, stenosis, duplication and malrotation) and gastrointestinal diseases (such as abdominal infection, necrotizing enterocolitis, intestinal obstruction, gastrointestinal perforation and meconium peritonitis). These individuals are a particularly susceptible population for candidemia, as they are often confronted with long-term fasting, and are more likely to undergo surgery and receive intravenous nutrition. Therefore in the present study, the differences in the symptoms, risk factors and prognosis for neonatal candidemia with or without gastrointestinal dysfunction were compared.

\section{Materials and methods}

Study design. The study was conducted at the Affiliated Children's Hospital of the Capital Institute of Pediatrics (Beijing, China), which is a 400-bed tertiary-level teaching 
hospital. The neonatal intensive care unit (NICU) is a nine-bed unit, which has between 750 and 800 admissions each year.

Patients. Candidemia was diagnosed through the use of blood cultures that yielded Candida species Sabouraud Dextrose Agar (SDA) medium (Jiangmen Jinzhang Science and Technology Development Co., Ltd., Tianjin, China) was used to identify the subspecies of Candida in neonates hospitalized in the NICU. The blood samples were collected from right and left femoral vein, or from right jugular vein and right femoral vein simultaneously. Case patients were identified through the records of the clinical microbiology laboratory at the Affiliated Children's Hospital of the Capital Institute of Pediatrics. The retrospective study included patients in the NICU who had positive cultures for Candida species, admitted between January 1, 2006 and December 31, 2010. Patients with positive results of Candida species from two cultural samples were allocated to the candidemia group, while individuals with negative results were placed in the non-candidemia group. The clinical characteristics, prognosis and previously identified risk factors for the two groups were recorded. The risk factors included gastrointestinal dysfunctions, the administration of antibiotics prior to the occurrence of candidemia, the presence of a central venous cannula, parenteral nutrition, gastrointestinal and secondary gastrointestinal surgery, repeated tracheal intubation and the occurrence of maternal candidal vaginitis. The outcomes and development situation at 6 months after discharge from hospital of the patient were also followed-up. The study was conducted in accordance with the Declaration of Helsinki and with approval from the Ethics Committee of the Affiliated Children's Hospital of the Capital Institute of Pediatrics. Written informed consent was obtained from parents or guardians of the participants. The patients were divided into gastrointestinal dysfunction and non-gastrointestinal dysfunction group, according to whether the patients were with or without congenital gastrointestinal dysfunction (gastrointestinal atresia, stenosis, duplication, malrotation, etc.) and gastrointestinal diseases (abdominal infection, necrotizing enterocolitis, intestinal obstruction, gastrointestinal perforation, meconium peritonitis, etc.).

Control patients. Control patients for the study were selected through the use of unit-specific patient admission databases. To increase the statistical efficiency, the individuals included in the non-candidemia and candidemia groups exhibited no statistically significant differences with regard to age and gender.

Statistical analysis. Categorical data were analyzed using the $\chi^{2}$ test, exact probability test and t-test. The Risk factors significantly related to candidemia according to the univariate analyses subsequently underwent multivariate analysis to identify the predictors following adjustment for possible comorbidities. $\mathrm{P}<0.05$ (two-tailed) was considered to indicate a statistically significant difference. Statistical analyses were performed using GraphPad Prism software, version 5 (GraphPad Software, Inc., San Diego, CA, USA). Patient age, length of hospital stay, length of ICU stay and treatment duration are presented as median. The enumeration data are expressed as percentage, relative risk, odds ratio and confidence interval.

\section{Results}

General information. During the study period, 19 case patients with candidemia were identified (male, 13; female, 6), while 21 patients (male, 14; female, 7) were included in the control group. The patients were aged between 1 and 28 days (median age, 13 days). Candidemia was as a result of Candida albicans in 10 patients $(52.63 \%)$, and due to non-Candida albicans species in 9 patients $(47.37 \%)$. The shortest hospitalization duration was 12 days, while the longest duration was 124 days (median duration, 28 days). The shortest duration in the ICU was 4 days and the longest was 49 days (median, 12 days). The shortest treatment duration was 0.42 weeks, while the longest was 8 weeks (median, 4 weeks). In total, $5 / 19$ patients $(26.32 \%)$ ultimately succumbed to their illness, of which three mortalities occurred in hospital (15.71\%) and two mortalities occurred within 90 days $(10.52 \%)$.

Clinical manifestations. Clinical manifestations of the candidemia group were more severe compared with the non-candidemia group (Table I). The results revealed that the symptoms of fever, abdominal pain, vomit, edema, serous effusion, tetter, thrush, shock, gastrointestinal and pulmonary hemorrhage and multiple organ failure were significantly more common in the candidemia group when compared with the non-candidemia group. Among the accompanying examination results, the occurrence of procalcitonin (PCT) levels of $>2 \mathrm{ng} / \mathrm{ml}$, hemoglobin levels of $<10 \mathrm{~g} / \mathrm{l}$, C-reactive protein (CRP) levels of $>50 \mathrm{mg} / \mathrm{l}$, thrombocytopenia, liver dysfunction, hypoproteinemia and pathogenic bloodstream infections other than Candida were significantly higher in the candidemia group compared with the non-candidemia group. In addition, the length of hospital stay and ICU stay in the candidemia group was significantly longer compared with the non-candidemia group (Fig. 1).

Risk factors. Comparisons between the candidemia and non-candidemia groups were conducted in terms of the presence of an underlying disease, risk factors and prognosis (Table II). A gastrointestinal dysfunction was the most common underlying disease in the patients from the candidemia group. According to the univariate analysis, the significant $(\mathrm{P} \leq 0.05)$ risk factors prior to the occurrence of candidemia in the candidemia group were antibiotic administration for $>2$ weeks $(P<0.0001)$, the use of more than three types of antibiotic $(\mathrm{P}=0.0019)$, the presence of a central venous cannula $(\mathrm{P}<0.0001)$, the use of parenteral nutrition for $>2$ weeks $(\mathrm{P}<0.0001)$, gastrointestinal surgery $(\mathrm{P}=0.0123)$, repeated tracheal intubation $(\mathrm{P}=0.0027)$, secondary gastrointestinal surgery and maternal candidal vaginitis $(\mathrm{P}=0.0424)$. When the prognosis of the two groups was compared, the proportion of patients with growth retardation 6 months after hospital discharge was significantly higher in the candidemia group $(\mathrm{P}=0.001)$. However, there was no statistically significant difference with regard to the hospital mortality rate and 90 -day mortality rate when comparing the two groups. Based on the forward step-wise multivariate regression analysis, the use of antibiotics for $>2$ weeks, maternal candidal vaginitis, repeated tracheal intubation and the use of glycopeptide antibiotics were demonstrated to be predictors of candidemia. 
Table I. Characteristics of the patients with or without Candida-positive cultures.

\begin{tabular}{|c|c|c|c|c|c|}
\hline Classification & $\begin{array}{l}\text { Candidemia, } \\
\mathrm{n}(\%)\end{array}$ & $\begin{array}{c}\text { Non-candidemia, } \\
\mathrm{n}(\%)\end{array}$ & P-value & RR $(95 \%$ CI $)$ & Odds ratio $(95 \% \mathrm{CI})$ \\
\hline \multicolumn{6}{|l|}{ Clinical feature } \\
\hline Fever & $13 / 19(68.42)$ & $3 / 21(14.28)$ & $0.0009^{c}$ & 4.78 (1.61-14.27) & $13.00(2.73-61.81)$ \\
\hline Abdominal distention & $13 / 19(68.42)$ & $2 / 21(9.52)$ & $0.0001^{\mathrm{c}}$ & $7.18(1.86-27.81)$ & $20.58(3.58-114.40)$ \\
\hline Vomiting & $12 / 19(63.15)$ & 2/21 (9.52) & $0.0004^{\mathrm{c}}$ & $6.63(1.70-25.91)$ & 16.29 (2.89-91.87) \\
\hline Edema & $11 / 19(57.89)$ & 4/21 (9.52) & $0.0211^{\mathrm{a}}$ & $3.04(1.16-7.95)$ & $5.84(1.41-24.18)$ \\
\hline Serous effusion & 8/19 (42.10) & 2/21 (9.52) & $0.0281^{\mathrm{a}}$ & $4.42(1.07-18.29)$ & $6.91(1.24-38.53)$ \\
\hline Tetter & $8 / 19(42.10)$ & $1 / 21(4.76)$ & $0.0072^{\mathrm{b}}$ & $8.84(1.22-64.33)$ & $14.55(1.60-132.00)$ \\
\hline Thrush & 8/19 (42.01) & 0/21 (0.00) & $0.0010^{c}$ & & $31.78(1.68-602.00)$ \\
\hline GI hemorrhage & $11 / 19(57.89)$ & $1 / 21(4.76)$ & $0.0003^{c}$ & $12.16(1.73-85.86)$ & $27.50(3.03-249.60)$ \\
\hline Pneumorrhagia & $5 / 19(26.31)$ & 0/21 (0.00) & $0.0177^{\mathrm{a}}$ & & $16.31(0.84-318.40)$ \\
\hline Shock & $10 / 19(52.63)$ & 2/21 (9.52) & $0.005^{\mathrm{b}}$ & $5.52(1.38-22.10)$ & $10.56(1.90-58.55)$ \\
\hline Multiple organ failure & 9/19 (47.36) & $1 / 21(4.76)$ & $0.0028^{\mathrm{b}}$ & $9.94(1.39-71.41)$ & $18.00(1.99-162.70)$ \\
\hline \multicolumn{6}{|l|}{ Accompanying examination } \\
\hline Leucocytes of $>20 \times 10^{9} / 1$ & $11 / 19(57.89)$ & 4/21 (19.05) & $0.0211^{\mathrm{a}}$ & $3.04(1.16-7.95)$ & $5.84(1.41-24.18)$ \\
\hline $\mathrm{Hb}$ of $<10 \mathrm{~g} / \mathrm{l}$ & $14 / 19(73.68)$ & $2 / 21(9.52)$ & $<0.0001^{\mathrm{c}}$ & $7.73(2.01-29.72)$ & $26.60(4.49-157.7)$ \\
\hline Thrombocytopenia & $14 / 19(73.68)$ & $3 / 21(14.28)$ & $0.0003^{c}$ & $5.16(1.75-15.22)$ & $16.80(3.41-82.64)$ \\
\hline PCT of $>2 \mathrm{ng} / \mathrm{ml}$ & $15 / 19(78.94)$ & $7 / 21(33.3)$ & $0.0051^{\mathrm{b}}$ & $2.37(1.24-4.53)$ & $7.50(1.78-31.29)$ \\
\hline CRP of >50 mg/l & $14 / 19(73.68)$ & 2/21 (9.52) & $<0.0001^{\mathrm{c}}$ & $7.73(2.01-29.72)$ & $26.60(4.49-157.70)$ \\
\hline Liver dysfunction & $14 / 19(73.68)$ & $3 / 21(14.28)$ & $0.0003^{c}$ & $5.16(1.75-15.22)$ & $16.80(3.41-82.64)$ \\
\hline Hypoproteinemia & $13 / 19(68.42)$ & 4/21 (19.05) & $0.0109^{\mathrm{a}}$ & $3.25(1.27-8.35)$ & $6.91(1.70-28.04)$ \\
\hline \multicolumn{6}{|l|}{ Other pathogens } \\
\hline Bloodstream infections & $10 / 19(52.63)$ & 2/21 (9.52) & $0.005^{\mathrm{b}}$ & $5.52(1.38-22.10)$ & $10.56(1.90-58.55)$ \\
\hline
\end{tabular}

${ }^{\mathrm{a}} \mathrm{P}=0.01-0.05,{ }^{\mathrm{b}} \mathrm{P}=0.001-0.01,{ }^{\mathrm{c}} \mathrm{P}<0.001$. Results are expressed as numbers and constituent ratios of patients. GI, gastrointestinal; Hb, hemoglobin; PCT, procalcitonin; CRP, C-reactive protein; RR, relative risk; CI, confidence interval.
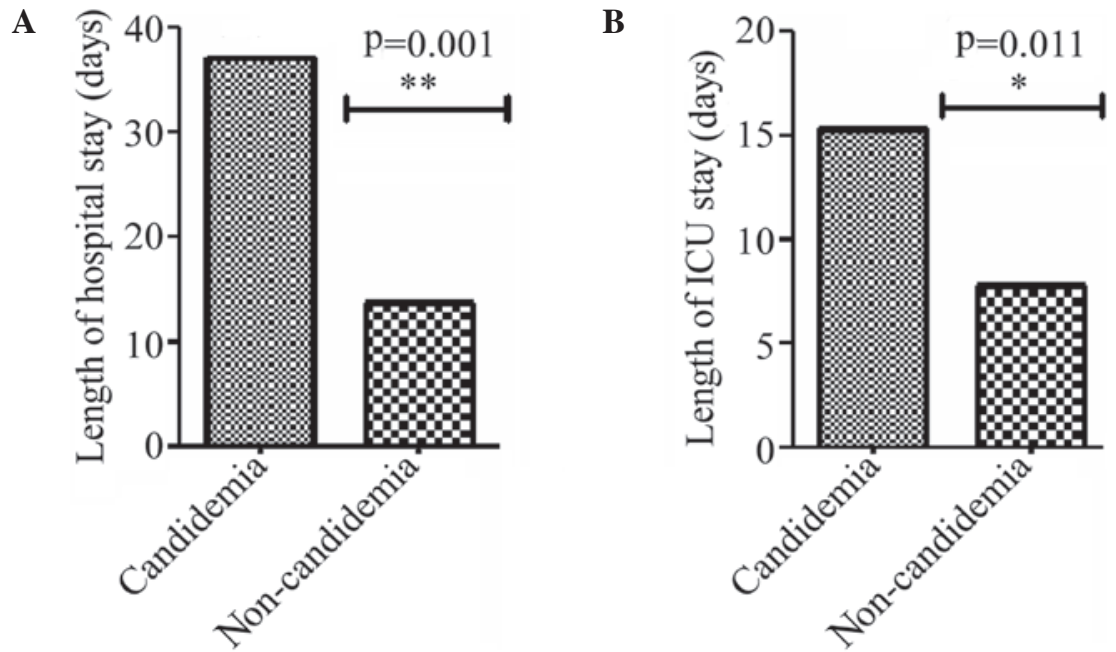

Figure 1. Length of stay in (A) hospital and (B) ICU for the candidemia and non-candidemia groups. ICU, intensive care unit.

Gastrointestinal dysfunction. In addition, differences in the symptoms, prognosis and risk factors of neonates with candidemia with or without gastrointestinal dysfunction were compared (Table III). Overall, $57.9 \%$ of the patients with candidemia exhibited gastrointestinal dysfunction. The incidence rates of shock, malnutrition, PCT levels of $>2 \mathrm{ng} / \mathrm{ml}$ and the presence of other pathogenic bloodstream infections were significantly higher in the gastrointestinal dysfunction group, whereas the incidence of premature birth and maternal candidal vaginitis was significantly higher in the non-gastrointestinal dysfunction group. The differences between the two groups with regard to the incidence rates of central venous 
Table II. Differences in the prognosis and risk factors of patients with or without Candida-positive cultures.

\begin{tabular}{|c|c|c|c|c|c|}
\hline Factors & Candidemia, $\mathrm{n}$ & Non-candidemia, $\mathrm{n}$ & P-value & RR $(95 \% \mathrm{CI})$ & Odds ratio $(95 \% \mathrm{CI})$ \\
\hline \multicolumn{6}{|l|}{ Underlying disease } \\
\hline GI dysfunctions & $11 / 19$ & $2 / 21$ & $0.0001^{\mathrm{b}}$ & $2.86(1.537-5.34)$ & $13.06(2.34-72.85)$ \\
\hline \multicolumn{6}{|l|}{ Risk factor } \\
\hline Prior use of antibiotics $>2$ weeks & $17 / 19$ & $1 / 21$ & $<0.0001^{\mathrm{c}}$ & $10.39(2.76-39.14)$ & $170(14.00-2044)$ \\
\hline$>3$ types of antibiotics & $11 / 19$ & $2 / 21$ & $0.0019^{\mathrm{b}}$ & $2.86(1.53-5.34)$ & $13.06(2.34-72.85)$ \\
\hline Carbapenems & $13 / 19$ & $3 / 21$ & $0.0009^{c}$ & $3.250(1.56-6.758)$ & $13(2.734-61.81)$ \\
\hline Glycopeptides & $10 / 19$ & $1 / 21$ & $0.001^{\mathrm{b}}$ & $2.93(1.65-5.20)$ & $22.22(2.46-200.90)$ \\
\hline Nitroimidazoles & $8 / 19$ & $1 / 21$ & $0.0072^{\mathrm{b}}$ & $2.51(1.48-4.25)$ & $14.55(1.60-132.00)$ \\
\hline Central venous cannula & $16 / 19$ & $2 / 21$ & $<0.0001^{\mathrm{c}}$ & $6.52(2.25-18.90)$ & $50.67(7.51-341.90)$ \\
\hline Parenteral nutrition $>2$ weeks & $16 / 19$ & $2 / 21$ & $<0.0001^{\mathrm{c}}$ & $6.52(2.25-18.90)$ & $50.67(7.51-341.90)$ \\
\hline GI surgery & $9 / 19$ & $2 / 21$ & $0.0123^{\mathrm{a}}$ & $2.37(1.33-4.21)$ & $8.55(1.54-47.43)$ \\
\hline Repeated endotracheal intubation & $7 / 19$ & $0 / 21$ & $0.0027^{\mathrm{b}}$ & $2.75(1.75-4.32)$ & $25.80(1.35-491.50)$ \\
\hline Secondary GI surgery & $7 / 19$ & $0 / 21$ & $0.0027^{\mathrm{b}}$ & $2.75(1.75-4.32)$ & $25.80(1.35-491.50)$ \\
\hline Maternal candidal vaginitis & $4 / 19$ & $0 / 21$ & $0.0424^{\mathrm{a}}$ & $2.40(1.63-3.53)$ & $12.48(0.62-249.40)$ \\
\hline \multicolumn{6}{|l|}{ Prognosis } \\
\hline Hospital mortality & $3 / 19$ & $0 / 21$ & 0.098 & & $9.1(0.43-189.3)$ \\
\hline 90-day mortality & $5 / 19$ & $1 / 21$ & 0.056 & $5.53(0.71-43.18)$ & $7.14(0.75-68.01)$ \\
\hline $\begin{array}{l}\text { Growth retardation at } 6 \text { months } \\
\text { after discharge }\end{array}$ & $11 / 19$ & $1 / 21$ & $0.001^{\mathrm{b}}$ & $2.93(1.65-5.20)$ & $22.22(2.46-200.90)$ \\
\hline
\end{tabular}

${ }^{\mathrm{a}} \mathrm{P}=0.01-0.05,{ }^{\mathrm{b}} \mathrm{P}=0.001-0.01,{ }^{\mathrm{C}} \mathrm{P}<0.001 . \mathrm{RR}$, relative risk; $\mathrm{CI}$, confidence interval; GI, gastrointestinal.

catheterization, intravenous nutrition, gastrointestinal surgery, repeated intubation, secondary gastrointestinal surgery and nitroimidazole and glycopeptide antibiotic administration were statistically significant $(\mathrm{P}<0.05)$. When comparing the prognosis of the two groups, who were followed-up for 6 months, all the patients with gastrointestinal dysfunction suffered growth retardation. A total of $72.2 \%$ of the patients in the gastrointestinal dysfunction group received mechanical ventilation, whereas $25 \%$ of the patients in the non-gastrointestinal dysfunction group received mechanical ventilation $(\mathrm{P}=0.0397)$. In addition, a total of $63.63 \%$ of patients in the gastrointestinal dysfunction group, compared with $12.5 \%$ of patients in the non-gastrointestinal dysfunction group, were effectively treated by removal of the central venous cannula, and the difference was statistically significant $(\mathrm{P}=0.0258)$. However, there was no statistically significant difference with regard to the efficacy of fluconazole, the utilization rate of human immunoglobulin, albumin or packed red cells between the two groups.

\section{Discussion}

The present study is a case-controlled study that examined the clinical features and risk factors for candidemia among neonates. Non-specific symptoms, such as fever, abdominal pain, vomiting, edema, serous effusion and tetter, were found to be the main clinical manifestations of candidemia. In addition, in certain cases, thrush may be a specific sign for neonates with severe candidemia. The high rates of shock, gastrointestinal and pulmonary hemorrhage and multiple organ failure in the patients with candidemia indicated that the neonates with candidemia were in a more severe clinical situation compared with the neonates without candidemia. Candida species can affect neonates more easily compared with adults and children, and usually damage multiple organ systems with increasing PCT and CRP levels when bloodstream infections occurred. As a result, patients with candidemia require longer hospital and ICU durations and expensive medical treatments.

Previous studies that have described risk factors for neonatal candidemia, in particular premature neonates. Saiman et al (8) reported limited data regarding the risk factors for candidemia, including gestational age of $<32$ weeks, 5-min Apgar score of $<5$, shock, disseminated intravascular coagulopathy, prior use of intralipids, parenteral nutrition, central venous catheters, $\mathrm{H}_{2}$ blockers, intubation and length of hospital stay of $>7$ days prior to candidemia. Yu et al (9) reported that the predominant risk factors for invasive fungal infections were third-generation cephalosporin administration, peripherally inserted central venous catheters, intubation of $>6$ days, any prior abdominal surgery and neutropenia $\left(<1.5 \times 10^{9}\right.$ cells $\left./ 1\right)$ during the first week of life. Furthermore, Pasqualotto et al (10) demonstrated that a failure to remove the central venous catheter was an independent risk factor for early mortality among pediatric patients with candidemia. Therefore, a number of risk factors for candidiasis in neonates have been reported, which were also observed in the patients included in the present study. Although the univariate analysis performed in the current study indicated that parenteral nutrition, tracheal intubation and central venous catheters were associated with candidemia, these associations were not statistically significant in the multivariate model. In the present study, independent risk factors for developing candidemia were evaluated. The results demonstrated that prior use of antibiotics for 
Table III. Differences in the symptoms, prognosis and risk factors of candidemia patients with or without GI dysfunctions.

\begin{tabular}{|c|c|c|c|c|c|}
\hline Factors & $\begin{array}{c}\text { GI } \\
\text { dysfunctions, } n\end{array}$ & $\begin{array}{c}\text { No GI } \\
\text { dysfunctions, n }\end{array}$ & $\chi^{2}$ & P-value & $\begin{array}{l}\text { Odds ratio } \\
(95 \% \mathrm{CI})\end{array}$ \\
\hline \multicolumn{6}{|l|}{ Underlying diseases } \\
\hline Premature birth & $2 / 11$ & $5 / 8$ & 3.909 & $0.048^{\mathrm{a}}$ & $0.13(0.02-1.09)$ \\
\hline \multicolumn{6}{|l|}{ Symptom } \\
\hline Malnutrition & $8 / 11$ & $2 / 8$ & 4.232 & $0.0397^{\mathrm{a}}$ & $8.00(1.00-64.00)$ \\
\hline Shock & $8 / 11$ & $2 / 8$ & 4.232 & $0.0397^{\mathrm{a}}$ & $8.00(1.00-64.00)$ \\
\hline \multicolumn{6}{|l|}{ Accessory examination } \\
\hline PCT of $>2 \mathrm{ng} / \mathrm{ml}$ & $10 / 11$ & $4 / 8$ & 3.997 & $0.0456^{\mathrm{a}}$ & $10.00(0.84-119.40)$ \\
\hline Other pathogenic bloodstream infections & $9 / 11$ & $3 / 8$ & 3.909 & $0.048^{\mathrm{a}}$ & $7.50(0.921-61.08)$ \\
\hline \multicolumn{6}{|l|}{ Risk factor } \\
\hline Parenteral nutrition & $11 / 11$ & $5 / 8$ & 4.868 & $0.027^{\mathrm{a}}$ & $0.07(0.00-1.57)$ \\
\hline Central venous catheter & $11 / 11$ & $5 / 8$ & 4.868 & $0.027^{\mathrm{a}}$ & $14.64(0.64-335.90)$ \\
\hline GI surgery & $9 / 11$ & $0 / 8$ & 12.44 & $0.0004^{\mathrm{c}}$ & $64.60(2.70-1546.0)$ \\
\hline Secondary GI surgery & $5 / 11$ & $0 / 8$ & 4.935 & $0.0263^{\mathrm{a}}$ & $14.38(0.67-310.10)$ \\
\hline Repeated endotracheal intubation & $7 / 11$ & $1 / 8$ & 4.958 & $0.0258^{\mathrm{a}}$ & $12.25(1.08-139.10)$ \\
\hline Nitroimidazoles & $8 / 11$ & $0 / 8$ & 10.05 & $0.0015^{\mathrm{b}}$ & $41.29(1.83-928.20)$ \\
\hline Glycopeptides & $8 / 11$ & $2 / 8$ & 4.232 & $0.0397^{\mathrm{a}}$ & $8.00(1.00-64.00)$ \\
\hline Maternal candidal vaginitis & $1 / 11$ & $4 / 8$ & 3.997 & $0.0456^{\mathrm{a}}$ & $0.10(0.00-1.19)$ \\
\hline \multicolumn{6}{|l|}{ Prognosis } \\
\hline Growth retardation at 6 months after discharge & $9 / 9$ & $2 / 7$ & 9.351 & $0.0022^{\mathrm{b}}$ & $0.024(0.00-0.59)$ \\
\hline \multicolumn{6}{|l|}{ Treatment } \\
\hline Mechanical ventilation & $8 / 11$ & $2 / 8$ & 4.232 & $0.0397^{\mathrm{a}}$ & $8.00(1.00-64.00)$ \\
\hline Removal of the central venous catheter & $8 / 11$ & $0 / 8$ & 10.05 & $0.0015^{\mathrm{b}}$ & $41.29(1.84-928.60)$ \\
\hline
\end{tabular}

${ }^{\mathrm{a}} \mathrm{P}=0.01-0.05,{ }^{\mathrm{b}} \mathrm{P}=0.001-0.01,{ }^{\mathrm{c}} \mathrm{P}<0.001 . \mathrm{CI}$, confidence interval; PCT, procalcitonin; GI, gastrointestinal.

$>2$ weeks, maternal candidal vaginitis, repeated gastrointestinal surgery and the prior use of glycopeptide antibiotics were associated with the development of candidemia in neonates.

A number of previous studies have investigated the association between antibiotic use and candidemia (11-13). In addition, numerous animal studies have demonstrated that normal anaerobic gastrointestinal flora provide an important defense mechanism against infection by inhibiting the growth of potentially pathogenic organisms (14-16). The translocation of yeast across an intact gastrointestinal mucosa via persorption has been demonstrated following the injection of Candida suspensions into a normal gastrointestinal tract (17). However, the presence of anaerobic bacteria in the gut has been clearly established to inhibit the overgrowth of Candida species $(18,19)$. A previous study (7) reported that administration of antimicrobials with activity against the anaerobic gastrointestinal flora is associated with the development of candidemia. Furthermore, the present analysis revealed that the administration of glycopeptide antibiotics was an independent risk factor for candidemia, which was not unexpected since glycopeptide antibiotics possess $\mathrm{N}$-acetyl glucosamine and mannose oligosaccharides capable of binding to mannnose-binding lectin which may decrease the clearance of significant pathogens such as yeast (20). The normal gastrointestinal barrier serves a crucial function in preventing Candida species colonizing the gastrointestinal tract and spreading into the blood. Therefore, the destruction of the gastrointestinal barrier by gastrointestinal surgery was a crucial risk factors for candidemia.

Additionally, differences in the symptoms, prognosis and risk factors among the patients in the candidemia group with and without gastrointestinal dysfunctions were compared. Infants with gastrointestinal diseases or malformations are more likely to require gastrointestinal surgery, which may result in an increased risk of candidemia due to the increased application of parenteral nutrition, antibiotic administration and a central venous catheter. To a certain extent, repeated tracheal intubation is associated with secondary gastrointestinal surgery. Prematurity has been reported as an important risk factor for neonatal candidemia (21). The results of the present study indicated that premature birth is a risk factor for candidemia in neonates without a gastrointestinal dysfunction. Newborns may have a higher risk of developing invasive candidiasis if their mother suffered from candidal vaginitis during perinatal period. Research has shown that isolates of Candida in the blood stream, the oral cavity of neonates and the vagina of the mother share a common genotype, which provides direct evidence of the association between congenital candidiasis in the neonate and candidal vaginitis in the mother (22). Furthermore, the present study observed that 4 newborns with candidemia had been associated with maternal candidal vaginitis during the perinatal period, which is rarely reported. 
With regard to prognosis, the results of the present study demonstrate that the incidence of growth retardation was higher in candidemia group compared with the non-candidemia group at 6 months after discharge from hospital, particularly in the gastrointestinal dysfunction group. A disorder in gastrointestinal function may be the primary cause of long-term malnutrition and growth retardation; therefore, the long-term prognosis of such infants should be followed-up closely.

In conclusion, candidemia threaten the life of neonates, in addition to influencing the early development of survivors. The clinical manifestations of candidemia usually involve multiple systems, and may resemble septic shock. Prior administration of antibiotics for $>2$ weeks, the use of glycopeptide antibiotics, maternal candidal vaginitis and secondary gastrointestinal surgery were identified as predictors of neonatal candidemia by multivariate analysis. In addition, gastrointestinal dysfunction appeared to be a crucial factor for the development of candidemia during the neonatal period. Further studies are required to identify additional risk factors for candidemia in critically ill neonates.

\section{References}

1. Maródi L and Johnston RB Jr: Invasive Candida species disease in infants and children: Occurrence, risk factors, management and innate host defense mechanisms. Curr Opin Pediatr 19: 693-697, 2007.

2. Zaoutis T: Candidemia in children. Curr Med Res Opin 26: 1761-1768, 2010.

3. Zaoutis TE, Argon J, Chu J, Berlin JA, Walsh TJ and Feudtner C: The epidemiology and attributable outcomes of candidemia in adults and children hospitalized in the United States: A propensity analysis. Clin Infect Dis 41: 1232-1239, 2005.

4. Cugno C and Cesaro S: Epidemiology, risk factors and therapy of candidemia in pediatric hematological patients. Pediatr Rep 4 e9, 2012.

5. Filioti J, Spiroglou K, Panteliadis CP and Roilides E: Invasive candidiasis in pediatric intensive care patients: Epidemiology, risk factors, management and outcome. Intensive Care Med 33: 1272-1283, 2007.

6. Ariff S, Saleem AF, Soofi SB and Sajjad R: Clinical spectrum and outcomes of neonatal candidiasis in a tertiary care hospital in Karachi, Pakistan. J Infect Dev Ctries 5: 216-223, 2011.

7. Zaoutis TE, Prasad PA, Localio AR, et al: Risk factors and predictors for candidemia in pediatric intensive care unit patients: Implications for prevention. Clin Infect Dis 51: e38-e45, 2010.
8. Saiman L, Ludington E, Pfaller M, et al: Risk factors for candidemia in neonatal intensive care unit patients. The National Epidemiology of Mycosis Survey study group. Pediatr Infect Dis J 19: 319-324, 2000 .

9. Yu Y, Du L, Yuan T, et al: Risk factors and clinical analysis for invasive fungal infection in neonatal intensive care unit patients. Am J Perinatol 30: 589-594, 2013.

10. Pasqualotto AC, de Moraes AB, Zanini RR and Severo LC: Analysis of independent risk factors for death among pediatric patients with candidemia and a central venous catheter in place. Infect Control Hosp Epidemiol 28: 799-804, 2007.

11. Spiliopoulou A, Dimitriou G, Jelastopulu E, Giannakopoulos I, Anastassiou ED and Christofidou M: Neonatal intensive care unit candidemia: Epidemiology, risk factors, outcome and critical review of published case series. Mycopathologia 173: 219-228, 2012.

12. Juyal D, Sharma M, Pal S, Rathaur VK and Sharma N: Emergence of non-albicans Candida species in neonatal candidemia. N Am J Med Sci 5: 541-545, 2013.

13. Vogiatzi L, Ilia S, Sideri G, et al: Invasive candidiasis in pediatric intensive care in Greece: A nationwide study. Intensive Care Med 39: 2188-2195, 2013.

14. Lawley TD and Walker AW: Intestinal colonization resistance. Immunology 138: 1-11, 2013.

15. Lamousé-Smith ES, Tzeng A and Starnbach MN: The intestinal flora is required to support antibody responses to systemic immunization in infant and germ free mice. PLoS One 6: e27662, 2011

16. de Moreno de LeBlanc A and LeBlanc JG: Effect of probiotic administration on the intestinal microbiota, current knowledge and potential applications. World J Gastroenterol 20: 16518-16528, 2014.

17. Shan YS, Sy ED, Wang ST, Lee JC and Lin PW: Early presumptive therapy with fluconazole for occult Candida infection after gastrointestinal surgery. World J Surg 30: 119-126, 2006.

18. Almirante B and Pemán J: Current treatment of candidemia. Role of anidulafungin. Enferm Infecc Microbiol Clin 26 (Suppl 14): 21-28, 2008 (In Spanish).

19. Garsin DA and Lorenz MC: Candida albicans and Enterococcus faecalis in the gut: Synergy in commensalism? Gut Microbes 4: 409-415, 2013.

20. Sealy PI, Garner B, Swiatlo E, Chapman SW and Cleary JD: The interaction of mannose binding lectin (MBL) with mannose containing glycopeptides and the resultant potential impact on invasive fungal infection. Med Mycol 46: 531-539, 2008.

21. Brenuchon C, Lebas D, Rakza T, Piette F, Storme L and Catteau B: Invasive fungal dermatitis in extremely premature newborns: A specific clinical form of systemic candidiasis. Ann Dermatol Venereol 133: 341-346, 2006.

22. Chen CJ, Weng YH, Su LH and Huang YC: Molecular evidence of congenital candidiasis associated with maternal candidal vaginitis. Pediatr Infect Dis J 25: 655-656, 2006. 